\title{
Critical magnetization behavior of the triangular- and kagome-lattice quantum antiferromagnets
}

\author{
Tôru Sakai ${ }^{1,2,3, *}$ and Hiroki Nakano ${ }^{2}$ \\ ${ }^{1}$ Japan Atomic Energy Agency, SPring-8, Sayo, Hyogo 679-5148, Japan \\ ${ }^{2}$ Graduate School of Material Science, University of Hyogo, Kouto 3-2-1, Kamigori, Ako-gun, Hyogo 678-1297 Japan \\ ${ }^{3}$ TRIP JST, Saitama 332-0012, Japan
}

(Received 17 December 2010; published 11 March 2011)

\begin{abstract}
We investigate $S=1 / 2$ quantum spin antiferromagnets on the triangular and Kagome lattices in a magnetic field, using the numerical exact diagonalization. We focus particularly on an anomalous magnetization behavior of each system at one-third the saturation magnetization. Critical exponent analyses suggest that it is a conventional magnetization plateau on the triangular lattice, while an unconventional phenomenon, called the magnetization ramp, occurs on the Kagome lattice.
\end{abstract}

DOI: 10.1103/PhysRevB.83.100405

PACS number(s): 75.10.Jm, 75.30.Kz, 75.40.Cx, 75.45.+j

$S=1 / 2$ triangular- and Kagome-lattice ${ }^{1}$ antiferromagnets have attracted a lot of interest as typical frustrated systems. Most theoretical studies have indicated that the former system has three-sublattice long-range order, ${ }^{2-4}$ while the latter is disordered in the ground state. ${ }^{5-16}$ Experimental studies to observe a novel spin liquid phase have been accelerated since discoveries of several realistic materials: the organic compound $\kappa$-(BEDT-TTF $)_{2} \mathrm{Cu}_{2}(\mathrm{CN})_{3}$ for the triangular lattice ${ }^{17}$ and herbertsmithite, ${ }^{18,19}$ volborthite, ${ }^{20,21}$ and vesignieite ${ }^{22}$ for the Kagome lattice. Since quantum Monte Carlo simulation and DMRG calculation are useless for these systems, numerical exact diagonalization is one of the best numerical methods for them. Numerical diagonalization studies suggest that both systems have the one-third magnetization plateau, ${ }^{23-27}$ although classical spin systems have no plateau on either lattice in the ground state. ${ }^{28,29}$ (Thermal or quantum fluctuations induce a plateau in the semiclassical case, because one-third is just a critical point between two different spin structures.) In our recent numerical diagonalization study of $S=1 / 2$ Kagome-lattice antiferromagnets up to $N=36$, the calculated field derivatives reveal an anomalous behavior at one-third the saturation magnetization. ${ }^{30}$ Namely, the field derivative diverges on the low-field side of the critical field $H_{c}$, while it is almost 0 on the high-field side. This critical behavior is quite different from conventional magnetization plateaus in twodimensional systems, where the field derivative is finite on both sides of $H_{c}$. To distinguish such an anomalous property at onethird magnetization of the Kagome lattice from conventional plateaus, we called it a "magnetization ramp." However, its mechanism is still an open problem. In this paper, to clarify this unconventional behavior around one-third magnetization of $S=1 / 2$ Kagome-lattice antiferromagnets, compared with the triangular one, we applied numerical diagonalization for both systems up to $N=39$, which is the largest cluster at present. In addition, we estimated the critical exponent $\delta$ by the finite-size scaling proposed in the previous work $^{32}$ to investigate the quantum critical behavior more quantitatively.

Now we examine the magnetization process of $S=1 / 2$ triangular- and Kagome-lattice antiferromagnets. The Hamiltonian is given by

$$
\begin{gathered}
\mathcal{H}=\mathcal{H}_{0}+\mathcal{H}_{Z} \\
\mathcal{H}_{0}=\sum_{\langle i, j\rangle} \mathbf{S}_{i} \cdot \mathbf{S}_{j}, \quad \mathcal{H}_{Z}=-H \sum_{j}^{N} S_{j}^{z}
\end{gathered}
$$

where $\langle i, j\rangle$ means all the nearest-neighbor pairs on each lattice. Throughout we use units such that $g \mu_{B}=1$. For $N$-site systems, the lowest energy of $\mathcal{H}_{0}$ in the subspace where $\sum_{j} S_{j}^{z}=M$ (macroscopic magnetization is $m=M / M_{\mathrm{s}}$, where $M_{\mathrm{s}}$ denotes saturation of the magnetization, namely, $M_{\mathrm{s}}=N S$ for a spin- $S$ system $)$ is denoted $E(N, M)$. We restrict ourselves to the rhombic cluster under the periodic boundary condition to maintain $120^{\circ}$ rotational symmetry for systematic finite-size scaling. Using numerical exact diagonalization, we have calculated all the values of $E(N, M)$ available for rhombic clusters with $N=9,12,21,27,36$, and 39 , to obtain the ground-state magnetization curves. (The largest dimension of the $N=39$ system is $68,923,264,410$. To treat such huge matrices in computers, we have carried out parallel calculations using the MPI-parallelized code which was originally developed in a previous work. ${ }^{31}$ ) Figure 1(a) and 1(b) show the magnetization curves of triangular- and Kagome-lattice antiferromagnets for $N=27,36$, and 39 . They indicate plateau-like behaviors at $m=1 / 3$ of both systems, but the Kagome lattice exhibits an anomalous feature; the step length increases with decreasing $H$ toward $m=$ $1 / 3$, different from conventional magnetization plateaus like triangular lattices. To clarify a difference between triangular and Kagome lattices, we calculated the field derivative $\chi$ defined in the form

$$
\chi^{-1}=\frac{E(N, M+1)-2 E(N, M)+E(N, M+1)}{1 / M_{\mathrm{s}}} .
$$

Figures 2(a) and 2(b) show the derivative $\chi$ of triangular- and Kagome-lattice systems, respectively, for $N=27,36$, and 39. The derivative $\chi$ of the triangular system is finite at both edges of the one-third plateau-like behavior, as in conventional twodimensional systems. In contrast, the Kagome system exhibits a quite different feature between the lower and the higher field sides of $m=1 / 3 ; \chi$ diverges on the lower side like a plateau in one-dimensional systems, while it is very small (possibly 0 ) on the higher side. The present calculation for $N=39$ more strongly supports the ramp-like behavior predicted by our previous work.

The critical exponent $\delta$ defined in the form

$$
\left|m-m_{c}\right| \sim\left|H-H_{c}\right|^{1 / \delta}
$$

is an important index for specifying the universality class of the field-induced quantum phase transition. Previous 

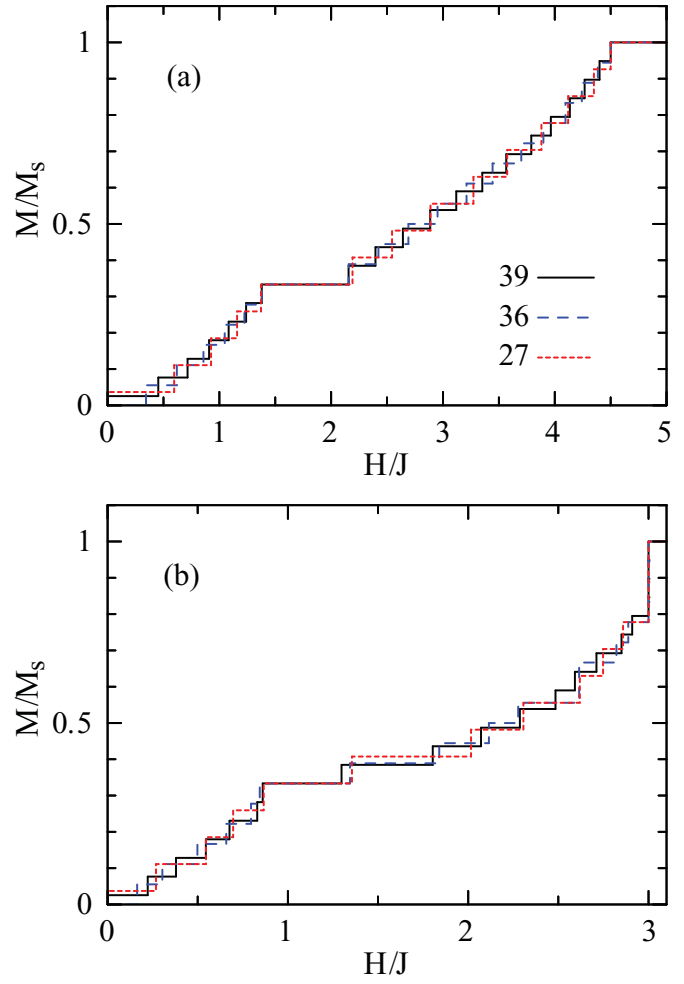

FIG. 1. (Color) Magnetization curves of (a) triangular- and (b) Kagome-lattice antifferomagnets for $N=27,36$, and 39.

theoretical work indicated $\delta=2$ for some typical onedimensional gapped systems, ${ }^{33,34}$ while $\delta=1$ for twodimensional systems. ${ }^{35}$ To investigate the quantum critical
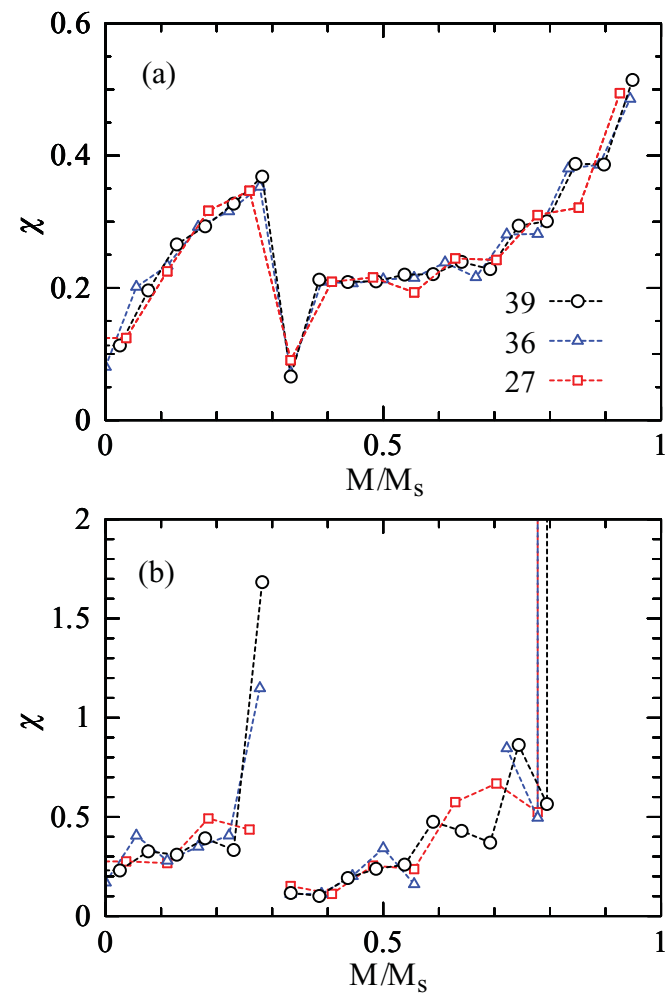

FIG. 2. (Color) Field derivatives of (a) triangular- and (b) Kagome-lattice antifferomagnets for $N=27,36$, and 39. behavior at $m=1 / 3$ of triangular- and Kagome-lattice antiferromagnets more quantitatively, we estimate $\delta$ by the finite-size scaling developed in a previous work. ${ }^{32}$ Although it was proposed for one-dimensional systems, it can easily be generalized for two dimensions. We assume the asymptotic form of the size dependence of the energy to be

$$
\frac{1}{N} E(N, M) \sim \epsilon(m)+C(m) \frac{1}{N^{\theta}} \quad(N \rightarrow \infty),
$$

where $\epsilon(m)$ is the bulk energy and the second term describes the leading size correction. We also assume that $C(m)$ is an analytic function of $m$. The lowest and highest magnetic fields corresponding to $m=1 / 3$ in the thermodynamic limit are defined as $H_{c-}$ and $H_{c+}$, respectively, in the form

$$
E\left(N, \frac{N}{3} \pm 1\right)-E\left(N, \frac{N}{3}\right) \rightarrow \pm H_{c \pm} \quad(N \rightarrow \infty) .
$$

To consider the critical magnetization behaviors for $m<1 / 3$ and $m>1 / 3$ independently, we define the critical exponents $\delta_{c-}$ and $\delta_{c+}$ by the forms

$$
\left|m-\frac{1}{3}\right| \sim\left|H-H_{c \pm}\right|^{1 / \delta_{ \pm}} .
$$

If we define the quantities $f_{+}(N)$ and $f_{-}(N)$ by the forms

$$
f_{ \pm}(N) \equiv \pm\left[E\left(N, \frac{N}{3} \pm 2\right)+E\left(N, \frac{N}{3}\right)-2 E\left(N, \frac{N}{3} \pm 1\right)\right]
$$

the asymptotic forms of them are expected to be

$$
f_{ \pm}(N) \sim \frac{1}{N^{\delta_{ \pm}}}+O\left(\frac{1}{N^{\theta+1}}\right) \quad(N \rightarrow \infty),
$$

as long as we assume the form (6). Thus the exponents $\delta_{-}$ and $\delta+$ can be estimated from the slope of the $\ln f_{ \pm}-\ln N$ plot, respectively, under the condition $\theta>\delta_{ \pm}-1$. To avoid an oscillation of the finite-size correction due to the cluster shape dependence, we just use rhombic clusters under the periodic boundary condition with $N=9,12,21,27,36$, and 39. Figures 3(a) and 3(b) show plots of $\ln f_{ \pm}$versus $\ln N$ for triangular- and Kagome-lattice antiferromagnets. Figure 3(a) suggests that the calculated points are well fitted to a line for each of $f_{-}$and $f_{+}$in the case of the triangular lattice. Thus applying the standard least squares fitting to lines (dashed and long-dashed lines are used to obtain $\delta_{+}$and $\delta_{-}$, respectively.) for all the available system sizes, $N=9,12,21,27,36$, and $39\left(N=9\right.$ cannot be used for $\left.\delta_{-}\right), \delta_{-}$and $\delta_{+}$are estimated as

$$
\delta_{-}=1.00 \pm 0.17, \quad \delta_{+}=0.89 \pm 0.15
$$

for the triangular lattice. Errors are estimated from the deviation of points from the fitted lines. It would be reasonable to conclude that $\delta_{-}=\delta_{+}=1$ at $m=1 / 3$ of the triangular-lattice antiferromagnet, as expected for conventional magnetization plateaus in two dimensions. In contrast, Fig. 3(b) indicates quite different features of the Kagome-lattice antiferromagnet. The same least squares fitting yields the estimates

$$
\delta_{-}=1.92 \pm 0.99, \quad \delta_{+}=0.56 \pm 0.15
$$

for the Kagome-lattice antiferromagnet. Exponent $\delta_{-}$has a large error because the line fitting is not good. It does not 

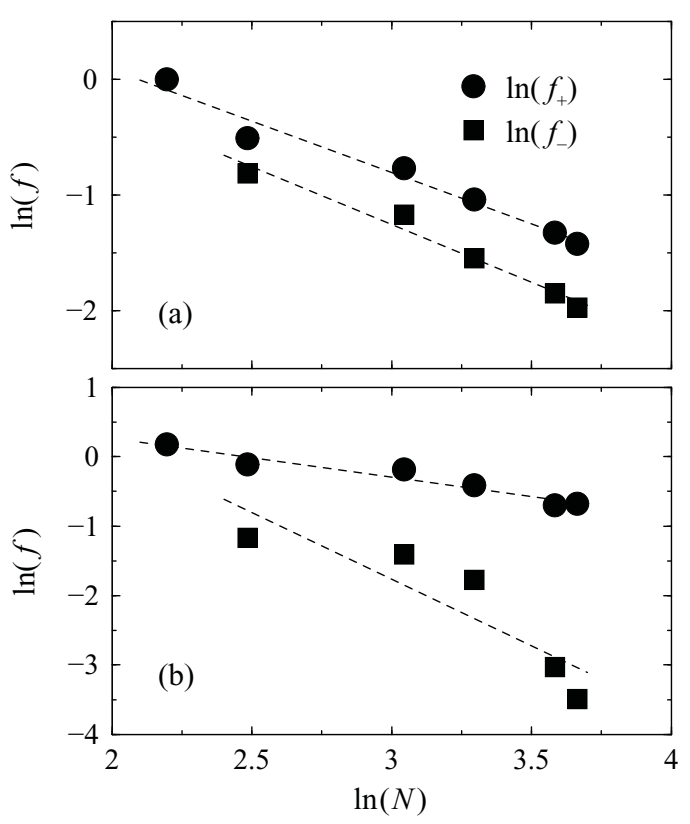

FIG. 3. $\ln (f)$ is plotted versus $\ln (N)$ for (a) triangular- and (b) Kagome-lattice antiferromagnets, respectively. Filled circles and squares represent estimated critical exponents $\delta+$ and $\delta-$, respectively.

converge well with respect to the system size but seems still to increase with $N$. The same line fitting to the points for $N=27,36$, and 39 yields the estimation $\delta_{-}=4.59 \pm 0.25$. Thus we can just conclude that $\delta_{-} \geqslant 2$ at most. This means that the divergent behavior of the field derivative at $H_{c-}$ is stronger than in one-dimensional systems. This leads to two possibilities. One is a jump (a first-order transition) in the magnetization curve. A magnetization jump that also appears near saturation was proved. ${ }^{36}$ The other possibility is an anomalous continuous transition. A similar phenomenon was reported in the metal-insulator transition of the Hubbard chain with next-nearest-neighbor hopping. ${ }^{37}$ In comparison with $\delta_{-}$, $\delta_{+}$is more conclusive, because the fitting error is much smaller. According to the above result of line fitting, we conclude that $\delta_{+}=0.6 \pm 0.2$. Thus the field derivative $\chi$ should be 0 on the higher field side of $H_{c+}$, because $\delta_{+}$is smaller than unity. It also justifies a property of the magnetization ramp.

Finally, we consider whether or not a flat part of the magnetization curve at $m=1 / 3$ exists for triangular- and Kagomelattice antiferromagnets. Namely, we examine whether each system has no plateau $\left(H_{c-}=H_{c+}\right)$ or a finite plateau $\left(H_{c-} \neq\right.$ $\left.H_{c+}\right)$ at $m=1 / 3$ in the thermodynamic limit. We evaluate the length of the flat part $H_{c+}-H_{c-}$ corresponding to the plateau width of finite-size clusters with $N=9,12,21,27$, 36 and 39 for both systems. If the system has a gapless excitation like a spin wave from some ordered states, the low-lying energy spectrum is expected to be proportional to the wave vector $k$ in the long-wavelength limit. Thus the excitation energy gap of the finite-size systems should have the asymptotic form $\sim 1 / N^{1 / 2}$ in two-dimensional gapless systems. In contrast, in gapped systems the gap is expected to converge to the thermodynamic limit with exponentially decaying (faster than $1 / N^{1 / 2}$ ) finite-size correction as the system size increases. Thus if extrapolation by fitting the gap

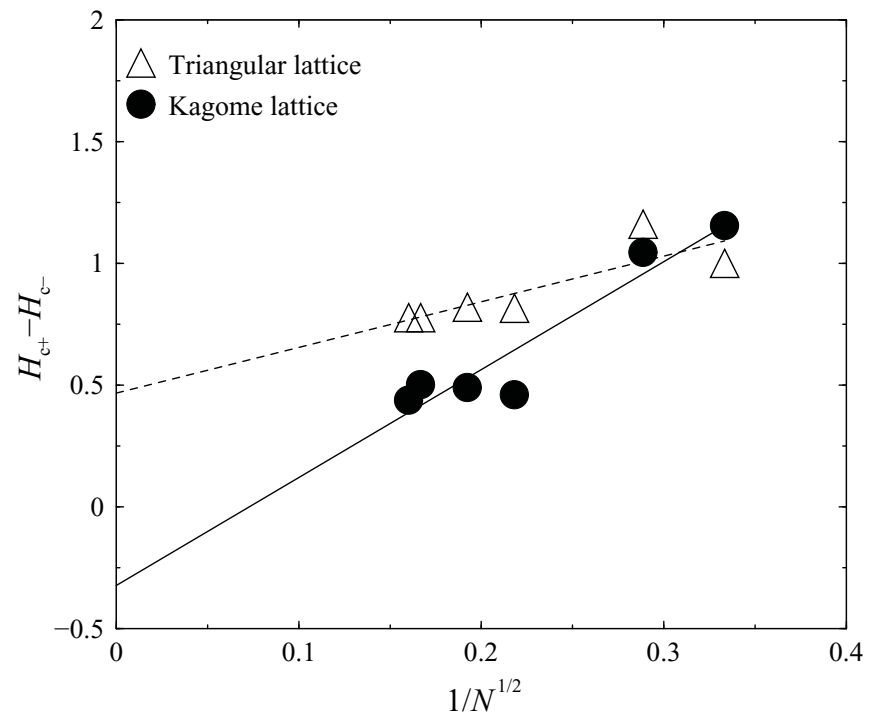

FIG. 4. Plateau width $H_{\mathrm{c}+}-H_{\mathrm{c}-}$ is plotted versus $1 / N^{1 / 2}$. Open triangles and filled squares represent triangular- and Kagome-lattice antiferromagnets, respectively. Fitted lines are used for extrapolation to the thermodynamic limit.

versus $1 / N^{1 / 2}$ leads to a finite gap in the thermodynamic limit, this would be strong evidence to confirm the gapped ground state. The length of the flat part $H_{c+}-H_{c-}$ is plotted versus $1 / N^{1 / 2}$ in Fig. 4 , where open triangles and filled circles are for triangular- and Kagome-lattice antiferromagnets, respectively. The least squares fitting to a line leads to the following results: $H_{c+}-H_{c-}=0.47 \pm 0.28$ for the triangular lattice and $H_{c+}-$ $H_{c-}=-0.32 \pm 0.35$ for the Kagome lattice. Obviously we can conclude that the triangular-lattice antiferromagnet has the one-third magnetization plateau. In contrast, the result for the Kagome lattice suggests that it possibly has a single critical field, $H_{c}=H_{c-}=H_{c+}$. However, it is difficult to exclude a finite magnetization plateau, because of the large error of the extrapolation. Note that any other plateaus are difficult to investigate using the present method, because fewer system sizes are available for $m \neq 1 / 3$.

In recent magnetization measurements ${ }^{20}$ of a candidate of the Kagome-lattice antiferromagnet volborthite, several steplike behaviors were observed, but they had not reached $m=$ $1 / 3$ yet. The same measurement is continuing to observe an anomaly at $m=1 / 3$, which is expected to be about $60 \mathrm{~T}$. It would be interesting to detect some unconventional features.

In summary, we have investigated critical magnetization behaviors at $m=1 / 3$ for $S=1 / 2$ triangular- and Kagomelattice quantum antiferromagnets, using numerical exact diagonalization of rhombic clusters up to $N=39$. The triangular lattice is revealed to have critical exponents $\delta_{-}=\delta_{+}=1$ and a finite plateau, which is consistent with a conventional magnetization plateau in two-dimensional systems. In contrast, the Kagome lattice is revealed to exhibit unconventional critical properties: $\delta_{-}<1<\delta_{+}$; that is, the field derivative $\chi$ is divergent on the lower field side, while it is 0 on the higher side of a possibly single critical field, $H_{c}=H_{c-}=H_{c_{+}}$. The conclusion supports magnetization ramp behavior at $m=1 / 3$ of the Kagome-lattice antiferromagnet. 
We wish to thank K. Hida and F. Mila for fruitful discussions. This work was partly supported by Grants-in-Aid Nos. 20340096 and 22014012 for Scientific Research and Priority Areas "Novel States of Matter Induced by Frustration" from the Ministry of Education, Culture, Sports, Science and Technology of Japan. Nonhybrid thread-parallel calculations in numerical diagonalizations were based on TITPACK version 2 coded by $\mathrm{H}$. Nishimori. Some of the computations were performed using the facilities of the Information Technology Center, Nagoya University, Department of Simulation Science, National Institute for Fusion Science, and Supercomputer Center, Institute for Solid State Physics, University of Tokyo. *sakai@ spring8.or.jp

${ }^{1} \mathrm{~A}$ brief history of the study of kagome-lattice magnetism was given by M. Mekata, Phys. Today, Feb. 12 (2003), [www.physicstoday.org].

${ }^{2}$ D. A. Huse and V. Elser, Phys. Rev. Lett. 60, 2531 (1988).

${ }^{3}$ B. Bernu, P. Lecheminant, C. Lhuillier, and L. Pierre, Phys. Rev. B 50, 10048 (1994).

${ }^{4}$ L. Capriotti, A. E. Trumper, and S. Sorella, Phys. Rev. Lett. 82, 3899 (1999).

${ }^{5}$ S. Sachdev, Phys. Rev. B 45, 12377 (1992).

${ }^{6}$ T. Nakamura and S. Miyashita, Phys. Rev. B 52, 9174 (1995).

${ }^{7}$ P. Lecheminant, B. Bernu, C. Lhuillier, L. Pierre, and P. Sindzingre, Phys. Rev. B 56, 2521 (1997).

${ }^{8}$ Ch. Waldtmann, H.-U. Everts, B. Bernu, C. Lhuillier, P. Sindzingre, P. Lecheminant, and L. Pierre, Eur. Phys. J. B 2, 501 (1998).

${ }^{9}$ F. Mila, Phys. Rev. Lett. 81, 2356 (1998).

${ }^{10}$ M. Hermele, T. Senthil, and M. P. A. Fisher, Phys. Rev. B 72, 104404 (2005).

${ }^{11}$ R. R. P. Singh and D. A. Huse, Phys. Rev. B 76, 180407(R) (2007).

${ }^{12}$ Y. Ran, M. Hermele, P. A. Lee, and X.-G. Wen, Phys. Rev. Lett. 98, 117205 (2007).

${ }^{13}$ H. C. Jiang, Z. Y. Weng, and D. N. Sheng, Phys. Rev. Lett. 98, 117203 (2007h).

${ }^{14}$ O. Cepas, C. M. Fong, P. W. Leung, and C. Lhuillier, Phys. Rev. B 78, 140405(R) (2008).

${ }^{15}$ P. Sindzingre and C. Lhuillier, Europhys. Lett. 88, 27009 (2009).

${ }^{16}$ G. Evenbly and G. Vidal, Phys. Rev. Lett. 104, 187203 (2010).

${ }^{17}$ Y. Shimizu, K. Miyagawa, K. Kanoda, M. Maesato, and G. Saito, Phys. Rev. Lett. 91, 107001 (2003).

${ }^{18}$ M. P. Shores, E. A. Nytko, B. M. Barlett, and D. G. Nocera, J. Am. Chem. Soc. 127, 13462 (2005).
${ }^{19}$ P. Mendels and F. Bert, J. Phys. Soc. Jpn. 79, 011001 (2010).

${ }^{20}$ H. Yoshida, Y. Okamoto, T. Tayama, T. Sakakibara, M. Tokunaga, A. Matsuo, Y. Narumi, K. Kindo, M. Yoshida, M. Takigawa, and Z. Hiroi, J. Phys. Soc. Jpn. 78, 043704 (2009).

${ }^{21}$ M. Yoshida, M. Takigawa, H. Yoshida, Y. Okamoto, and Z. Hiroi, Phys. Rev. Lett. 103, 077207 (2009).

${ }^{22}$ Y. Okamoto, H. Yoshida, and Z. Hiroi, J. Phys. Soc. Jpn. 78, 033701 (2009).

${ }^{23}$ K. Hida, J. Phys. Soc. Jpn. 70, 3673 (2001).

${ }^{24}$ D. C. Cabra, M. D. Grynberg, P. C. W. Holdsworth, and P. Pujol, Phys. Rev. B 65, 094418 (2002).

${ }^{25}$ J. Schulenburg, A. Honecker, J. Schnack, J. Richter, and H.-J. Schmidt, Phys. Rev. Lett. 88, 167207 (2002).

${ }^{26}$ A. Honecker, J. Schulenburg, and J. Richter, J. Phys. Condens. Matter 16, S749 (2004).

${ }^{27}$ D. C. Cabra, M. D. Grynberg, P. C. W. Holdsworth, A. Honecker, P. Pujol, J. Richter, J. Schmalfus, and J. Schulenburg, Phys. Rev. B 71, 144420 (2005)

${ }^{28}$ H. Kawamura and S. Miyashita, J. Phys. Soc. Jpn. 54, 4530 (1985).

${ }^{29}$ M. E. Zhitomirsky, Phys. Rev. Lett. 88, 057204 (2002).

${ }^{30}$ H. Nakano and T. Sakai, J. Phys. Soc. Jpn. 79, 053707 (2010).

${ }^{31}$ H. Nakano and A. Terai, J. Phys. Soc. Jpn. 78, 014003 (2009).

${ }^{32}$ T. Sakai and M. Takahashi, Phys. Rev. B 57, R8091 (1998).

${ }^{33}$ I. Affleck, Phys. Rev. B 43, 3215 (1991).

${ }^{34}$ T. Sakai and M. Takahashi, Phys. Rev. B 43, 13383 (1991); J. Phys. Soc. Jpn. 60, 3615 (1991).

${ }^{35}$ N. Katoh and M. Imada, J. Phys. Soc. Jpn. 63, 4529 (1994).

${ }^{36}$ M. E. Zhitomirsky and H. Tsunetsugu, Phys. Rev. B 70, 100403 (2004).

${ }^{37}$ H. Nakano and Y. Takahashi, J. Phys. Soc. Jpn. 73, 983 (2004). 